\title{
Strategy-proof cardinal decision schemes
}

\author{
Bhaskar Dutta • Hans Peters • Arunava Sen
}

Published online: 23 February 2008

(C) Springer-Verlag 2008

\section{Erratum to: Soc Choice Welfare (2007) 28:163-179 DOI 10.1007/s00355-006-0152-9}

As was first pointed out to us by John Hegeman, ${ }^{1}$ the proof of Claim 3, Theorem 1, in Dutta et al. (2007) is not correct, since it is based on interchanging two limits which is not justified without, for instance, a continuity assumption.

In this note we first give an alternative proof of $\lambda_{j} \leq \lambda_{j}^{\prime}$ (notations as in Dutta et al. (2007)).

To show this assume, to the contrary, $\lambda_{j}>\lambda_{j}^{\prime}$. By Claim 2 we can take $u_{1}$ with $\tau\left(u_{1}\right)=a_{j}$ and-for simplicity- $u_{1}(a)=0$ for all $a \neq a_{j}$. Let $0<\varepsilon<\frac{1}{2}\left(\lambda_{j}-\lambda_{j}^{\prime}\right)$ and let $\eta_{2}$ be so small that $\left|\varphi_{j}\left(u_{1}, u_{k j}^{\eta_{2}}\right)-\lambda_{j}^{\prime}\right|<\varepsilon$. Observe that agent 1's utility in this profile is equal to $\varphi_{j}\left(u_{1}, u_{k j}^{\eta_{2}}\right)$.

\footnotetext{
${ }^{1}$ Stanford University.
}

The online version of the original article can be found under doi:10.1007/s00355-006-0152-9.

\section{B. Dutta $(\varangle)$}

University of Warwick, Coventry, UK

e-mail: b.dutta@warwick.ac.uk

\section{H. Peters}

Department of Quantitative Economics, University of Maastricht, P.O. Box 616, 6200 MD Maastricht, The Netherlands e-mail: h.peters@ke.unimaas.nl

\section{A. Sen}

Indian Statistical Institute, New Delhi, India

e-mail: asen@isid.ac.in 
Next, take $\eta_{1}$ so small that $\left|\varphi_{j}\left(u_{j k}^{\eta_{1}}, u_{k j}^{\eta_{2}}\right)-\lambda_{j}\right|<\varepsilon$. This is possible in view of Claim 2 (with the roles of the agents there reversed). Then according to $u_{1}$ agent 1 's utility is now $\varphi_{j}\left(u_{j k}^{\eta_{1}}, u_{k j}^{\eta_{2}}\right)$, but $\varphi_{j}\left(u_{j k}^{\eta_{1}}, u_{k j}^{\eta_{2}}\right)>\varphi_{j}\left(u_{1}, u_{k j}^{\eta_{2}}\right)$. This violates strategyproofness.

Unfortunately, at this moment we do not know how to prove the reverse inequality $\lambda_{j} \geq \lambda_{j}^{\prime}$ and, thus, Claim 3, without making additional assumptions. One possibility would be to extend the set of admissible utility functions by dropping the requirement that there be a unique top alternative and assume continuity of the $\operatorname{CDS} \varphi$. Another possibility is to strengthen the unanimity condition by requiring that if every agent in a preference profile has the same two top alternatives, then all other alternatives should receive zero probability. A third possibility is to impose, additionally, the following requirement on $\varphi$, which is a kind of unanimity:

(*) For all admissible profiles $u$ and all $a_{j} \in A$, if $u_{i}\left(a_{k}\right) \geq u_{i}\left(a_{j}\right)$ for all $a_{k} \in$ $A \backslash\left\{a_{i}\right\}$ and $i \in N$, then $\varphi_{j}(u)=0$.

In other words, if the agents have a common bottom alternative, then that alternative should receive zero probability. We will prove $\lambda_{j} \geq \lambda_{j}^{\prime}$ under this additional assumption (*). To the contrary, assume $\lambda_{j}<\lambda_{j}^{\prime}$. Let $0<\varepsilon<\frac{1}{2}\left(\lambda_{j}^{\prime}-\lambda_{j}\right)$. By Claim 2, we may assume that the utility functions under consideration have a common bottom alternative $b$. Also by Claim 2, we may assume that $u_{j k}^{\eta_{1}}$ satisfies $u_{j k}^{\eta_{1}}(a)=1-\eta_{1}-\alpha\left(\eta_{1}\right)$ for all (if any) $a \neq a_{j}, a_{k}, b$, with $\alpha\left(\eta_{1}\right)>0$ satisfying

$$
\frac{\eta_{1}+\alpha\left(\eta_{1}\right)}{\eta_{1}}<\frac{1-\lambda_{j}-\varepsilon}{1-\lambda_{j}^{\prime}+\varepsilon}
$$

(observe that the latter expression is greater than 1).

Now let $u_{1}$ with $\tau\left(u_{1}\right)=a_{j}$ and let $\eta_{2}>0$ so small that $\left|\varphi_{j}\left(u_{1}, u_{k j}^{\eta_{2}}\right)-\lambda_{j}^{\prime}\right|<\varepsilon$. Next, let $\eta_{1}>0$ so small that $\left|\varphi_{j}\left(u_{j k}^{\eta_{1}}, u_{k j}^{\eta_{2}}\right)-\lambda_{j}\right|<\varepsilon$. Then player 1's utility (according to $u_{j k}^{\eta_{1}}$ ) from truthful reporting is smaller than

$$
\left(\lambda_{j}+\varepsilon\right)+\left(1-\lambda_{j}-\varepsilon\right)\left(1-\eta_{1}\right)
$$

whereas his utility from falsely reporting $u_{1}$ is greater than

$$
\left(\lambda_{j}^{\prime}-\varepsilon\right)+\left(1-\lambda_{j}^{\prime}-\varepsilon\right)\left(1-\eta_{1}-\alpha\left(\eta_{1}\right)\right)
$$

Note that for (3) we have used that the bottom alternative $b$ receives zero probability by (*). By (1) it follows that the expression in (3) is strictly greater than the expression in (2), which violates strategy-proofness.

At the moment we cannot show that $(*)$ nor any other of the mentioned alternative additional assumptions are redundant.

\section{Reference}

Dutta B, Peters H, Sen A (2007) Stategy-proof cardinal decision schemes. Soc Choice Welfare 28: 163-197 